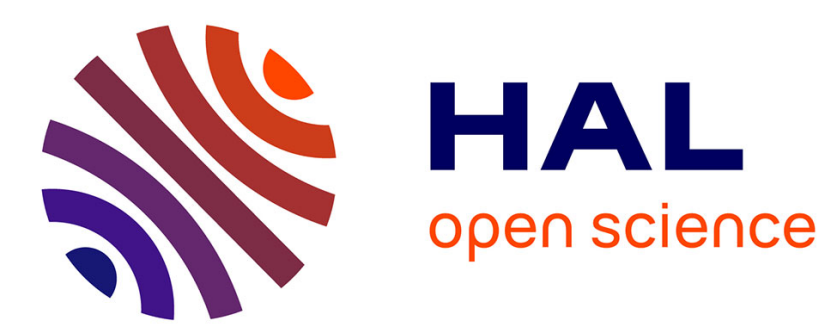

\title{
An existence result for polynomial solutions of parameter-dependent LMIs
}

\author{
Pierre-Alexandre Bliman
}

\section{To cite this version:}

Pierre-Alexandre Bliman. An existence result for polynomial solutions of parameter-dependent LMIs.

[Research Report] RR-4798, INRIA. 2003. inria-00071788

\section{HAL Id: inria-00071788 \\ https://hal.inria.fr/inria-00071788}

Submitted on 23 May 2006

HAL is a multi-disciplinary open access archive for the deposit and dissemination of scientific research documents, whether they are published or not. The documents may come from teaching and research institutions in France or abroad, or from public or private research centers.
L'archive ouverte pluridisciplinaire HAL, est destinée au dépôt et à la diffusion de documents scientifiques de niveau recherche, publiés ou non, émanant des établissements d'enseignement et de recherche français ou étrangers, des laboratoires publics ou privés. 
INSTITUT NATIONAL DE RECHERCHE EN INFORMATIQUE ET EN AUTOMATIQUE

\title{
An existence result for polynomial solutions of parameter-dependent LMIs
}

\author{
Pierre-Alexandre Bliman
}

$\mathbf{N}^{\circ} 4798$

Avril 2003

THÈME 4 



\title{
An existence result for polynomial solutions of parameter-dependent LMIs
}

\author{
Pierre-Alexandre Bliman* \\ Thème 4 - Simulation et optimisation \\ de systèmes complexes \\ Projet Sosso \\ Rapport de recherche $\mathrm{n}^{\circ} 4798$ - Avril $2003-6$ pages
}

\begin{abstract}
We show in this report that any system of Linear Matrix Inequalities depending continuously upon scalar parameters and solvable for any value of the latter in a fixed compact set, admits a branch of solutions polynomial with respect to the parameters. This result is useful for studying e.g. parametric robustness or gain-scheduling issues.
\end{abstract}

Key-words: Linear matrix inequalities, regularity of the solutions

* Email : pierre-alexandre.bliman@inria.fr. 


\section{Un résultat d'existence de solutions polynômiales pour les inégalités linéaires matricielles dépendant de paramètres}

Résumé : Nous montrons dans ce rapport que tout système d'inégalités linéaires matricielles dépendant continûment de paramètres scalaires et soluble pour toute valeur de ceux-ci dans un ensemble compact fixé, admet une branche de solutions polynômiales par rapport à ces paramètres. Ce résultat est utile pour l'étude par exemple de problèmes de robustesse paramètrique ou de séquencement de gains.

Mots-clés : Inégalités linéaires matricielles, régularité des solutions 


\section{Introduction}

Linear Matrix Inequalities (LMIs) have become a powerful unifying framework for expressing and solving many problems in control theory. This class of convex optimization problems, solved by efficient interior-point methods, has spread widely, in particular since the publication in 1994 of the by now classical monograph by S. Boyd et al. [4]. Among other, stability, stabilizability, detectability, $H^{2}$ and $H^{\infty}$ performance analysis, and various related design issues may be stated as LMIs, see e.g. recent progress in [8].

The next important step was to introduce parameter-dependent LMIs, see e.g. the exposition in [1] and the references therein. The latter appear naturally when studying control techniques robust against parametric uncertainty, or gain-scheduling methods, as these issues amount to check solvability of LMIs obtained for different values of some parameters. Generally, these parameters are a priori unknown but assumed to be inside a certain prespecified bounded set, and an attempt to extend the use of LMI solvers to these problems immediatly reaches an obstacle: the impossibility to check an infinite (usually uncountable) number of independent LMI conditions.

An early way to circumvent this difficulty has been to assume prescribed, simple, dependency of the LMI solutions with respect to the parameters, see references in [3] in the context of robust stability analysis. According to the admissible parameter set, in the case of constant or affine dependency, but also for general polynomial dependency [3], the coefficients of the involved polynomials may be found (for given value of the degree) as solutions of standard LMIs.

However, very few results exist, guaranteeing existence of solutions with prespecified dependency, to parameter-dependent LMIs. In other words, nothing is known in general on the conservatism of this simplifying assumption, and thus of the derived approaches. For the Lyapunov inequality $P=P^{T}>0$, $A^{T} P+P A<0$, for given parameter-dependent matrix $A$, use of the integral form of the Lyapunov equation $A^{T} P+P A=-I$ permits to show existence of a polynomial solution, in domains where $A$ is analytical and Hurwitz.

In [6, Lemma 1.1], Delchamps established an analyticity result, which permits to conclude that the LMIs which may be transformed into Riccati inequality by Schur transformation possess, if they are solvable, solutions polynomial with respect to their coefficients. Stated initially in the real case, this result is extended to the complex case in [9, Chapter 4, Lemma p. 134].

Last in [5, Proposition 2.1], Y.-S. Chou et al. provided a result ensuring existence of polynomial solution, for LMIs depending upon one complex parameter lying on the unit circle.

In the present short note, we provide a general result, Theorem 1, ensuring existence of polynomial solution to any solvable LMI depending continuously upon scalar parameters lying in a fixed compact set. Direct consequences are stated in Corollaries 2, 3. Applications of these results will be developed in further contributions.

\section{Main result}

We consider in the sequel the following property:

$$
\exists x \in \mathbb{R}^{p}, G(x, \delta) \stackrel{\text { def }}{=} G_{0}(\delta)+x_{1} G_{1}(\delta)+\cdots+x_{p} G_{p}(\delta)>0 .
$$

Here, $G_{0}, G_{1}, \ldots, G_{p}$ are mappings defined in a compact subset $K$ of $\mathbb{R}^{m}$, and taking values in the set of symmetric matrices of $\mathbb{R}^{n \times n}$. Formula (1) is to be seen as a parameter-dependent LMI, depending upon the parameter $\delta \in K$, and with unknown $x$. It represents indeed the general form of a LMI depending upon scalar parameters.

The aim of the present note is to establish the following result.

Theorem 1. Suppose $G_{0}, G_{1}, \ldots, G_{p}$ are continuous. If, for any $\delta \in K$, there exists $x(\delta) \in \mathbb{R}^{p}$ such that $G(x(\delta), \delta)>0$, then there exists a polynomial function $x^{*}: K \rightarrow \mathbb{R}^{p}$, such that, for any $\delta \in K$, $G\left(x^{*}(\delta), \delta\right)>0$.

The following result, on non-strict inequalities, is a straightforward consequence of Theorem 1.

RR $n^{\circ} 4798$ 
Corollary 2. Suppose $G_{0}, G_{1}, \ldots, G_{p}$ are continuous. Let $E$ be a continuous function, mapping $K$ into the set of positive definite symmetric matrices in $\mathbb{R}^{n \times n}$. If, for any $\delta \in K$, there exists $x(\delta) \in \mathbb{R}^{p}$ such that $G(x(\delta), \delta) \geq 0$, then there exists a polynomial function $x^{*}: K \rightarrow \mathbb{R}^{p}$, such that, for any $\delta \in K$, $G\left(x^{*}(\delta), \delta\right)>-E(\delta)$.

To prove Corollary 2, apply Theorem 1 to the LMI:

$$
\exists x \in \mathbb{R}^{p}, G(x, \delta)+E(\delta)>0 .
$$

Another direct consequence of Theorem 1 gives more informations on the structure of the solution set of the parameter-dependent LMI $G(x, \delta)>0$.

Corollary 3. Suppose $G_{0}, G_{1}, \ldots, G_{p}$ are continuous. Let $\underline{E}, \bar{E}$ be continuous functions, mapping $K$ into the set of symmetric matrices in $\mathbb{R}^{n \times n}$. If, for any $\delta \in K$, there exists $x(\delta) \in \mathbb{R}^{p}$ such that $\bar{E}(\delta)>$ $G(x(\delta), \delta)>\underline{E}(\delta)$, then there exists a polynomial function $x^{*}: K \rightarrow \mathbb{R}^{p}$, such that, for any $\delta \in K$, $\bar{E}(\delta)>G\left(x^{*}(\delta), \delta\right)>\underline{E}(\delta)$.

The proof of Corollary 3 consists in applying Theorem 1 to the LMI:

$$
\exists x \in \mathbb{R}^{p},\left(\begin{array}{cc}
G(x, \delta)-\underline{E}(\delta) & 0_{n} \\
0_{n} & \bar{E}(\delta)-G(x, \delta)
\end{array}\right)>0 .
$$

Proof of Theorem 1. Under the hypothesis of solvability of (1) for any $\delta \in K$, there exists, by continuity and compactness, a real number $\alpha>0$ such that

$$
\forall \delta \in K,\left\{x \in \mathbb{R}^{p}: G_{0}(\delta)+x_{1} G_{1}(\delta)+\cdots+x_{p} G_{p}(\delta) \geq 2 \alpha I_{n}\right\} \neq \emptyset .
$$

Define

$$
F: K \rightarrow 2^{\mathbb{R}^{p}}, \delta \mapsto F(\delta)=\left\{x \in \mathbb{R}^{p}: G_{0}(\delta)+x_{1} G_{1}(\delta)+\cdots+x_{p} G_{p}(\delta) \geq \alpha I_{n}\right\} .
$$

The set-valued map $F$ maps $K$ into the non-void closed convex subsets of $\mathbb{R}^{p}$.

Let us first establish that $F$ fulfils the following property of lower semicontinuity, see e.g. [2].

Definition. Let $X$ be a topological space, $Y$ a metric space. A set-valued map $F$ from $X$ to $Y$ is said lower semicontinuous at $x^{0} \in X$ if for any $y^{0} \in F\left(x^{0}\right)$ and any neighborhood $N\left(y^{0}\right)$ of $y^{0}$, there exists a neighborhood $N\left(x^{0}\right)$ such that

$$
\forall x \in N\left(x^{0}\right), F(x) \cap N\left(y^{0}\right) \neq 0 .
$$

$F$ is said lower semicontinuous if it is lower semicontinuous at every point $x^{0} \in X$.

Let $\delta^{0} \in K, x^{0} \in F\left(\delta^{0}\right), \varepsilon>0$. To prove lower semicontinuity of $F$ at $\delta^{0}$, we exhibit $\eta>0$ such that for any $\delta \in K$ with $\left\|\delta-\delta^{0}\right\|_{m}<\eta$, there exists $x \in F(\delta),\left\|x-x^{0}\right\|_{p}<\varepsilon$.

Indeed, by assumption, there exists $x^{\delta^{0}} \in \mathbb{R}^{p}$ such that $G\left(x^{\delta^{0}}, \delta^{0}\right) \geq 2 \alpha I_{n}$. For $\lambda \in[0,1]$ to be defined afterwards, let $x \stackrel{\text { def }}{=}(1-\lambda) x^{0}+\lambda x^{\delta^{0}}$. Then, the fact that $G$ is affine with respect to $x$ implies for any $\eta>0$, any $\delta \in K$ such that $\left\|\delta-\delta^{0}\right\|_{m}<\eta$ :

$$
\begin{aligned}
G(x, \delta) & =(1-\lambda) G\left(x^{0}, \delta\right)+\lambda G\left(x^{\delta^{0}}, \delta\right) \\
& =(1-\lambda) G\left(x^{0}, \delta^{0}\right)+\lambda G\left(x^{\delta^{0}}, \delta^{0}\right)+(1-\lambda)\left(G\left(x^{0}, \delta\right)-G\left(x^{0}, \delta^{0}\right)\right)+\lambda\left(G\left(x^{\delta^{0}}, \delta\right)-G\left(x^{\delta^{0}}, \delta^{0}\right)\right) \\
& \geq \alpha(1+\lambda) I_{n}-\left(\sup _{\left\|\delta-\delta^{0}\right\|_{m}<\eta}\left\|G\left(x^{0}, \delta\right)-G\left(x^{0}, \delta^{0}\right)\right\|_{n}+\sup _{\left\|\delta-\delta^{0}\right\|_{m}<\eta}\left\|G\left(x^{\delta^{0}}, \delta\right)-G\left(x^{\delta^{0}}, \delta^{0}\right)\right\|_{n}\right) I_{n} .
\end{aligned}
$$

On the other hand,

$$
\left\|x-x^{0}\right\|_{p}=\lambda\left\|x^{\delta^{0}}-x^{0}\right\|_{p} .
$$


So, take $\lambda \in[0,1]$ such that

$$
\lambda \leq \frac{\varepsilon}{2\left\|x^{\delta^{0}}-x^{0}\right\|_{p}}
$$

and choose $\eta>0$ such that

$$
\sup _{\left\|\delta-\delta^{0}\right\|_{m}<\eta}\left\|G\left(x^{0}, \delta\right)-G\left(x^{0}, \delta^{0}\right)\right\|_{n}+\sup _{\left\|\delta-\delta^{0}\right\|_{m}<\eta}\left\|G\left(x^{\delta^{0}}, \delta\right)-G\left(x^{\delta^{0}}, \delta^{0}\right)\right\|_{n} \leq \alpha \lambda .
$$

With these choices, one has $\left\|x-x^{0}\right\|_{p} \leq \varepsilon / 2<\varepsilon$, and $G(x, \delta) \geq \alpha(1+\lambda) I_{n}-\alpha \lambda I_{n}=\alpha I_{n}$, so $x \in F(\delta)$, provided that $\delta \in K$ and $\left\|\delta-\delta^{0}\right\|_{m}<\eta$. One concludes that $F$ is lower continuous at $\delta^{0}$. This achieves the proof of lower semicontinuity of $F$.

We now apply to $F$ defined in (2) Michael's Selection Theorem [10], see also [2].

Theorem (Michael's Selection Theorem). Let $X$ be a metric space, $Y$ a Banach space. Let $F$ from $X$ into the closed convex subsets of $Y$ be lower semicontinuous. Then there exists $f: X \rightarrow Y$, a continuous selection from $F$. that

This yields existence of a continuous selection $f: K \rightarrow \mathbb{R}^{p}$ from $F$ defined in (2). This function is such

$$
\forall \delta \in K, G(f(\delta), \delta) \geq \alpha I_{n}
$$

It remains to apply to each of the $p^{2}$ coefficients of $f$ the following result, see e.g. [7].

Theorem (Weierstrass Approximation Theorem). Every continuous real-valued function defined on a compact subset $K$ of $\mathbb{R}^{m}$, is the limit of a sequence of polynomials, which converges uniformly in $K$.

Thus, the selection $f$ previously exhibited is uniform limit in $K$ of a sequence of (matrix-valued) polynomials in $x$. In particular, there exists a polynomial function $x^{*}: K \rightarrow \mathbb{R}^{p}$ such that

$$
\forall \delta \in K, G\left(x^{*}(\delta), \delta\right) \geq \frac{\alpha}{2} I_{n}>0
$$

One concludes that there exists a polynomial solution to the parameter-dependent LMI (1), and this achieves the proof of Theorem 1 .

\section{Acknowledgement}

The author wishes to express his indebtedness to Jean-Pierre Quadrat, for valuable discussions on the topics exposed here.

\section{References}

[1] P. Apkarian, H.D. Tuan (2000). Parameterized LMIs in control theory, SIAM Journal on Control and Optimization 38 no 4, 1241-1264

[2] J.-P. Aubin, A. Cellina (1984). Differential inclusions. Set-valued maps and viability theory, SpringerVerlag, Berlin Heidelberg New-York Tokyo

[3] P.-A. Bliman (2002). Nonconservative LMI approach to robust stability for systems with uncertain scalar parameters, Proc. of 41st IEEE CDC, Las Vegas (Nevada)

[4] S. Boyd, L. El Ghaoui, E. Feron, V. Balakrishnan (1994). Linear Matrix Inequalities in System and Control Theory, SIAM Studies in Applied Mathematics vol. 15

RR $\mathrm{n}^{\circ} 4798$ 
[5] Y.-S. Chou, A.L. Tits, V. Balakrishnan (1999). Stability multipliers and $\mu$ upper bounds: connections and implications for numerical verification of frequency domain conditions, IEEE Trans. Automat. Control 44 no 5, 906-913

[6] D.F. Delchamps (1984). Analytic feedback control and the algebraic Riccati equation, IEEE Trans. Automat. Control AC-29 no 11, 1031-1033

[7] J. Dieudonné (1969). Foundations of modern analysis, Enlarged and corrected printing. Pure and Applied Mathematics Vol. 10-I, Academic Press, New-York London

[8] L. El Ghaoui, S.-I. Niculescu eds. (2000). Advances in Linear Matrix Inequality Methods in Control, SIAM Philadelphia

[9] E.W. Kamen (1985). Stabilization of linear spatially-distributed continuous-time and discrete-time systems, Multidimensional systems theory. Progress, directions and open problems in multidimensional systems, Mathematics and its Applications 16, D. Reidel Publishing Co, Dordrecht, N.K. Bose Ed., 101-146

[10] E. Michael (1956). Continuous selections I, Annals of Math. 63 361-381 


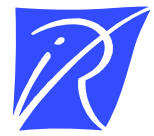

Unité de recherche INRIA Rocquencourt Domaine de Voluceau - Rocquencourt - BP 105 - 78153 Le Chesnay Cedex (France)

Unité de recherche INRIA Lorraine : LORIA, Technopôle de Nancy-Brabois - Campus scientifique 615, rue du Jardin Botanique - BP 101 - 54602 Villers-lès-Nancy Cedex (France)

Unité de recherche INRIA Rennes : IRISA, Campus universitaire de Beaulieu - 35042 Rennes Cedex (France)

Unité de recherche INRIA Rhône-Alpes : 655, avenue de l'Europe - 38330 Montbonnot-St-Martin (France)

Unité de recherche INRIA Sophia Antipolis : 2004, route des Lucioles - BP 93 - 06902 Sophia Antipolis Cedex (France)

INRIA - Domaine de Voluceau - Rocquencourt, BP 105 - 78153 Le Chesnay Cedex (France)

http://www.inria.fr

ISSN 0249-6399 